\title{
Audio-based Localization for Swarms of Micro Air Vehicles
}

\author{
Meysam Basiri $^{1,2}$, Felix Schill ${ }^{1}$, Dario Floreano ${ }^{1}$ and Pedro U.Lima ${ }^{2}$
}

\begin{abstract}
Localization is one of the key challenges that needs to be considered beforehand to design truly autonomous MAV teams. In this paper, we present a cooperative method to address the localization problem for a team of MAVs, where individuals obtain their position through perceiving a soundemitting beacon MAV that is flying relative to a reference point in the environment. For this purpose, an on-board audio-based localization system is proposed that allows individuals to measure the relative bearing to the beacon robot and furthermore to localize themselves and the beacon robot simultaneously, without the need for a communication network. Our method is based on coherence testing among signals of a small on-board microphone array, to obtain the relative bearing measurements, and an estimator, to fuse these measurements with sensory information about the motion of the robot throughout time, to estimate robustly the MAV positions. The proposed method is evaluated both in simulation and in real world experiments.
\end{abstract}

\section{INTRODUCTION}

Employing a swarm of autonomous robots, for achieving tasks in a collaborative manner, has been of great interest in the field of robotics. Teams of micro air vehicles (MAVs) can accomplish aerial coverage tasks more robustly and more efficiently compared to a single flying robot. Tasks such as security patrols or searching for victims inside a disaster area can benefit from several autonomous MAVs operating in parallel. In addition, by employing multiple MAVs and by sharing resources among them, it is potentially possible to use teams of low cost, lightweight and safe Micro Air Vehicles (MAVs) instead of a large and expensive aerial platform. Other possible applications of MAV swarms include rapidly-deployable communication networks [1], environmental monitoring, aerial surveillance and mapping, traffic monitoring and search and rescue [2].

A key challenge in designing truly autonomous MAVs is the robot localization problem, that is the problem of estimating the MAV's location relative to its environment. Individual's knowledge about their 3D position is essential for allowing MAVs to navigate autonomously to different points in space and to achieve aerial coverage tasks such as exploration and mapping. This information could also be used in multi-MAV systems to avoid inter robot collisions by priori spatial separation of individuals at different altitudes or locations [3]. Furthermore, by sharing their position with other team members, individuals can obtain the relative

\footnotetext{
*This work was supported by a doctoral grant from FCT (SFRH/BD/51070/2010), EC FP-7 research funding mechanism under grant agreement no. 266470 and FCT project [PEst-OE/EEI/LA0009/2013].

1 Laboratory of Intelligent Systems, Ecole Polytechnique Federale de Lausanne, CH-1015 Lausanne, Switzerland (e-mail: meysam.basiri, felix.schill, dario.floreano@epfl.ch)

2 Institute for Systems and Robotics, Instituto Superior Tecnico, Lisboa, Portugal (e-mail: pal@isr.ist.utl.pt)
}

position of their neighbouring robots and use this information to form formations [4] [5] and avoid collisions with other MAVs without the need of priori spatial separation [6].

A localization system for a MAV must satisfy strict constraints in terms of weight, size, power consumption, processing power, three-dimensional coverage and price. These constraints limit the use of many successful localization systems, used on ground robots or large aerial vehicles, for MAVs. Inspired by some animal groups [7], [8], which use sound for localization, we propose an audio-based localization system that allows individuals in a MAV group to obtain their absolute location, by only measuring the relative bearing to a beacon MAV that is emitting a bird-like chirp sound throughout time. An audio-based localization system satisfies the imposed constraints on MAVs and furthermore have the advantage of being independent of illumination, weather conditions, such as fog, smoke and dust, and possible occlusions caused by obstacles or other MAVs. In previous works we showed the success of using sound to locate acoustic targets on the ground [2] and to obtain the relative position between members of a multi-MAV system [9]. Such systems could potentially be exploited to perceive other non-cooperative noise emitting aerial platforms.

This paper is organized as follows: Section II describes the related work on localization systems for MAVs. Section III describes our proposed method for MAV localization in a Multi-MAV system, by firstly explaining the estimator that localizes the MAVs when bearing-only measurements are available, in Subsection III-A, and furthermore explaining the on-board audio-based relative bearing measurement system, in Subsection III-B. Section IV provides the results of simulation and real world experiments of the proposed method.

\section{STATE OF THE ART}

In general, robot localization methods that are addressed in the literature can be divided into two main categories:

1) Global Localization methods

2) Local Localization methods

Methods based on the former approach determine the absolute position of robots relative to a global reference frame with the assist of an external system. Using external Colour vision cameras or infrared 3-D motion tracking cameras [10], for indoor aerial robots, and using Global Positioning System (GPS) and wireless positioning beacons [11], for outdoor aerial robots, are examples of methods in this category. The advantage with solutions based on this approach is the accuracy that they usually provide while having a low computational complexity. The main drawback is their dependency on an external system that is not always 
available. Deployment of wireless positioning beacons in the environment and in advance of each mission, if not impossible, is both costly and time-consuming. Furthermore, GPS technologies are vulnerable to jamming and interferences, have low resolution, and are impossible to use in cluttered environments where there is no direct line of sight with the transmitting satellites [12]. GPS vulnerability is considered as one of the main problems that need to be solved before allowing MAVs to operate in civilian airspace [13].

Due to disadvantages of the first approach, effort has been put into the design of local localization methods, where the position of robots are obtained locally, using onboard sensors, and independent of any external systems. In this group of methods, localization is achieved using probabilistic techniques and by only employing on-board proprioceptive and exteroceptive sensor information. The most common examples of this approach are the vision based SLAM (Simultaneous Localization and Mapping) algorithms that mainly use an onboard camera to map features in the environment and to localize the robot [14], [15], [16]. A drawback with the local localization methods is that they mainly require a high computational power and a high data storage for operation. This might not always be available, specially on small scale micro air vehicles. The need for real-time processing of high resolution and high frame-rate images, the dependency on illumination, visual contrast, weather conditions and the limited field of view of vision sensors, the errors caused due to the high or insufficient number of features in the images, the long displacement between loop closings and the fast dynamic nature of MAVs, are some of the major drawbacks of the visual SLAM methods for aerial robots [16].

\section{PROPOSED METHOD}

This section explains our method for localizing MAVs in a Multi-MAV system. This method can be considered as a combination between the two class of approaches, as discussed in Section II, to employ some of the advantages from both classes. It allows a group of MAVs to cooperatively localize themselves using only their onboard sensors, and without the need for any external systems, while avoiding the high complexity nature of the local localization methods. The idea here is that a single MAV in the group starts to fly in a circular pattern around a static reference point and furthermore acts as a positioning beacon attracting the attention of other MAVs in the group. Other MAVs then measure the relative bearing to this beacon robot and estimate the position of both the beacon robot and themselves, throughout time, relative to the reference point. No communication between the robots is required and only prior knowledge of the behaviour of the beacon robot is used in the estimations.

As illustrated in Figure 1, the beacon robot is controlled to circle around a desired reference point, while trying to maintain a previously defined altitude, speed and circling radius. Many control strategies for guiding a MAV on a circular path exists [17], [18]. In this work, a vector field based controller, similar to the one proposed in [18], was used to control the motion of the beacon MAV around the

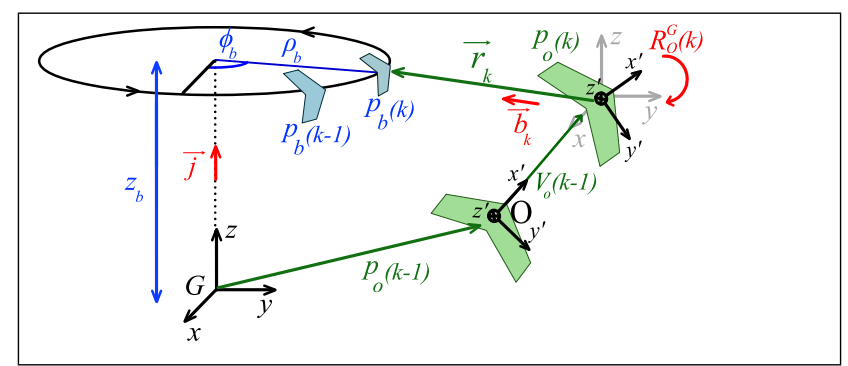

Fig. 1. Diagram illustrating the positions of two MAVs, beacon MAV $\left(p_{b}\right)$ and observing MAV $\left(p_{o}\right)$, for two successive time steps.

reference point. The beacon MAV can consider a static point on the ground, detected by an onboard camera [19], or a static acoustic target on the ground, detected by an onboard microphone array [2], as the reference point.

\section{A. Bearing-only localization}

In this section, an Extended Kalman Filtering (EKF) based estimator is derived to provide an observing MAV with a robust estimate of its location. This is achieved by fusing the noisy relative bearing measurements with information about the motion of the MAV through out time, given by its onboard proprioceptive sensors, and taking into account the prior knowledge about the behaviour of the beacon robot. The estimator is recursive and consists of an Initialization step and two iterative steps, Prediction and Update, that are explained in the following subsections.

1) Extended Kalman Filtering (EKF):

At time instant $k$, the position of the beacon MAV and an observing MAV, relative to the reference point, is given by position vectors $p_{b}(k)$ and $p_{o}(k)$ respectively, where $p_{b}$ is defined in Cylindrical coordinate system by $p_{b}=\left(\rho_{b}, \phi_{b}, z_{b}\right)$ and $p_{o}$ is defined in Cartesian coordinate system by $p_{o}=$ $\left(x_{o}, y_{o}, z_{o}\right)$. The combination of both position vectors is considered as the state vector $X$ for the EKF:

$$
X=\left(\begin{array}{c}
p_{b} \\
p_{o}
\end{array}\right)=\left[\begin{array}{llllll}
\rho_{b} & \phi_{b} & z_{b} & x_{o} & y_{o} & z_{o}
\end{array}\right]^{T}
$$

Furthermore, a $6 \times 6$ covariance matrix $P(k)$ defines the state error covariance matrix at time instant $k$.

Initialization: In this work, an initial state estimation strategy is proposed in order to obtain a good initial guess of the state vector $X(0)$ to have a faster convergence in the state estimation. The EKF is initialized after the first reliable bearing measurement is obtained, by using the MAV's orientation and altitude sensor values:

$$
\begin{aligned}
p_{b}(0) & =\left(R_{b}, 0, Z_{b}\right) \\
p_{o}(0) & =Z_{b} \vec{j}-\ell\left(R_{O}^{G} \overrightarrow{b_{0}}\right) \\
\ell & =\left\{\begin{array}{cc}
\frac{\left(Z_{b}-Z_{o}\right)}{\operatorname{sign}\left(Z_{b}-Z_{o}(0)\right) \vec{j} \cdot R_{O}^{G}(0) \overrightarrow{b_{0}}} & Z_{b} \neq Z_{o} \\
\frac{D_{M}}{2} & Z_{b}=Z_{o}
\end{array}\right.
\end{aligned}
$$

where, $Z_{b}$ and $R_{b}$ are the prior knowledge of the beacon MAV's altitude and circling radius respectively, $\vec{j}$ is a unit vector along the positive $z$ axis of the global coordinate system $G,(\cdot)$ is the vector dot product, $Z_{o}(0)$ is the measured altitude of the observer MAV at time zero and $D_{M}$ is the 
maximum detection range of the bearing measuring sensor. Vector $\overrightarrow{b_{0}}$ is a unit vector in the observer MAV's body fixed coordinate system $O$ pointing along the direction of the initial relative bearing measurement. $R_{O}^{G}(0)$ is a rotation matrix that rotates vectors from coordinate system $O$ to $G$ :

$$
R_{O}^{G}(k)=R_{z}\left(-\lambda_{o}(k)\right) R_{y}\left(-\beta_{o}(k)\right) R_{x}\left(-\alpha_{o}(k)\right)
$$

$\left(\lambda_{o}(k), \beta_{o}(k), \alpha_{o}(k)\right)$ are the yaw, roll and pitch orientation measurements of the observer robot and $\left(R_{z}, R_{y}, R_{x}\right)$ are basic rotation matrices that rotate vectors about the local $z, y, x$ axis respectively. In the case of ideal measurements and when $Z_{b} \neq Z_{o}$, equation (3), obtained using basic vector operations, calculates the center point of a circle of radius $R_{b}$ that have the observer MAV on its circumference. Furthermore, a covariance matrix $P(0)$ is initialized:

$$
P(0)=\operatorname{diag}\left(\sigma_{\rho_{b}(0)}^{2}, \sigma_{\phi_{b}(0)}^{2}, \sigma_{z_{b}(0)}^{2}, \sigma_{x_{o}(0)}^{2}, \sigma_{y_{o}(0)}^{2}, \sigma_{z_{o}(0)}^{2}\right)
$$

where $\sigma_{x(0)}^{2}$ is the initial covariance of the state variable $x$ that are chosen in accordance to the reliability of sensor readings and the uncertainties in the initial state estimation.

Prediction: In the prediction step, the current state of the system $\tilde{X}(k)$ is predicted from $X(k-1)$. For the observer MAV, a probabilistic motion model and the onboard sensor information, providing the speed and orientation of the MAV, is used to predict the position vector $\tilde{p}_{o}(k)$ from $p_{o}(k-1)$.

$$
\tilde{p}_{o}(k)=p_{o}(k-1)+R_{O}^{G}(k-1)\left[\begin{array}{c}
V_{o}(k-1) d t \\
0 \\
0
\end{array}\right]
$$

where $V_{o}(k)$ is the speed sensor reading and $d t$ is the time interval between the two time steps. The motion model (5) is derived by assuming that, at every iteration, the MAV has a forward motion along the $\mathrm{x}$-axis of its body fixed coordinate system, followed by a three dimensional rotation.

If communication between the robots were available, the speed and orientation values of the beacon MAV along with conversions between Cylindrical and Cartesian coordinate systems could also be used to predict the beacon MAV's position vector $\tilde{p}_{b}(k)$. However, as we are interested in a solution that does not depend on a communication network, only the prior knowledge about the speed $V_{b}$ and circling radius $R_{b}$ is used to obtain $\tilde{p}_{b}(k)$ :

$$
\tilde{p}_{b}(k)=p_{b}(k-1)+\left[\begin{array}{ccc}
0 & \frac{V_{b}}{R_{b}} d t & 0
\end{array}\right]^{T}
$$

Furthermore, a prediction of the state covariance matrix, $\tilde{P}(k)$, is obtained by assuming that the uncertainty in state predictors (5) and (6) is a zero mean multivariate Gaussian.

Update: In the Update step, the relative bearing measurement, presented by vector $\overrightarrow{b_{k}}$, is used to update the state prediction $\tilde{X}(k)$. For this, a measurement model to predict the relative bearing from the state predictions is defined:

$$
\theta=\tan ^{-1}\left(\frac{r_{y}}{r_{x}}\right) \quad \varphi=\tan ^{-1}\left(\frac{r_{z}}{\sqrt{r_{x}^{2}+r_{y}^{2}}}\right)
$$

where

$$
\vec{r}=\left(r_{x}, r_{y}, r_{z}\right)=\left(\rho_{b} \cos \phi_{b}-x_{o}, \rho_{b} \sin \phi_{b}-y_{o}, z_{b}-z_{o}\right)
$$

is a vector in the global coordinate system $G$ that starts at the position $p_{o}$ and ends at the position $p_{b} . \theta$ and $\varphi$ are the azimuth and elevation of vector $\vec{r}$. The predicted bearing $(\tilde{\theta}$, $\tilde{\varphi})$ is found by substituting the state predictions $\tilde{X}(k)$, from equations (5) and (6), into equation (7).

Furthermore, an innovation $\mu(k)$ is defined as the difference between the predicted bearing $\left(\tilde{\theta}_{k}, \tilde{\varphi}_{k}\right)$ and the measured bearing $\left(\hat{\theta}_{k}, \hat{\varphi}_{k}\right)$ :

$$
\mu(k)=\left[\begin{array}{cc}
\hat{\theta}_{k}-\tilde{\theta}_{k} & \hat{\varphi}_{k}-\tilde{\varphi}_{k}
\end{array}\right]^{T}
$$

where $\hat{\theta}_{k}$ and $\hat{\varphi}_{k}$ are the azimuth and elevation of vector $\overrightarrow{b_{k}}$ expressed in the coordinate system $G$, i.e $R_{O}^{G}(k) \overrightarrow{b_{k}}$. The innovation covariance matrix $S(k)$ is computed by:

$$
S(k)=H \tilde{P}(k) H^{T}+D
$$

where $D$ is the error covariance of bearing measurements and is found empirically. $H$ is the Jacobian of the measurement model (7) with respect to the states:

$$
H=\left.\left[\begin{array}{c}
\frac{\partial \theta}{\partial X} \\
\frac{\partial \varphi}{\partial X}
\end{array}\right]\right|_{\tilde{X}(k)}=\left.\left[\begin{array}{ccc}
H_{11} & \ldots & H_{16} \\
H_{21} & \ldots & H_{26}
\end{array}\right]\right|_{\tilde{X}(k)}
$$

where

$$
\begin{aligned}
& H_{11}=\left(y_{o} \cos \varphi_{b}-x_{o} \sin \varphi_{b}\right) / \Re_{1} \\
& H_{12}=\rho_{b}\left(\rho_{b}-x_{o} \cos \varphi_{b}-y_{o} \sin \varphi_{b}\right) / \Re_{1} \\
& H_{14}=\left(-y_{o}+\rho_{b} \sin \varphi_{b}\right) / \Re_{1} \\
& H_{15}=\left(x_{o}+\rho_{b} \cos \varphi_{b}\right) / \Re_{1} \\
& H_{13}=H_{16}=0 \\
& H_{21}=\left(\left(z_{o}-z_{b}\right)\left(\rho_{b}-x_{o} \cos \varphi_{b}-y_{o} \sin \varphi_{b}\right)\right) / \Re_{3} \\
& H_{22}=-\rho_{b}\left(z_{o}-z_{b}\right)\left(y_{o} \cos \varphi_{b}-x_{o} \sin \varphi_{b}\right) / \Re_{3} \\
& H_{24}=\left(z_{o}-z_{b}\right)\left(x_{o}-\rho_{b} \cos \varphi_{b}\right) / \Re_{3} \\
& H_{25}=\left(z_{o}-z_{b}\right)\left(y_{o}-\rho_{b} \sin \varphi_{b}\right) / \Re_{3} \\
& H_{23}=-H_{26}=\sqrt{\Re_{1}} / \Re_{2} \\
& \Re_{1}=\rho_{b}^{2}+x_{o}^{2}+y_{o}^{2}-2 \rho_{b}\left(x_{o} \cos \varphi_{b}+y_{o} \sin \varphi_{b}\right) \\
& \Re_{2}=\Re_{1}+\left(z_{o}-z_{b}\right)^{2} \\
& \Re_{3}=\Re_{2} \sqrt{\left(x_{o}-\rho_{b} \cos \varphi_{b}\right)^{2}+\left(y_{o}-\rho_{b} \sin \varphi_{b}\right)^{2}}
\end{aligned}
$$

Finally the states are updated:

$$
\begin{aligned}
& X(k)=\tilde{X}(k)+K(k) \mu(k) \\
& P(k)=\tilde{P}(k)-K(k) H \tilde{P}(k)
\end{aligned}
$$

where $K(k)$ is the Kalman gain at time $k$ derived by:

$$
K(k)=\tilde{P}(k) H^{T} S(k)^{-1}
$$

\section{B. Audio-based relative bearing measurement}

The previous section described a method of estimating the $3 \mathrm{D}$ position of members in a swarm of MAVs when only relative-bearing measurements to a flying beacon MAV is available. In this section, the proposed sensor suite for obtaining these measurements is described. Figure 2 shows the schematic diagram of the on-board audio-based relative bearing measurement system, describing its key units.

The beacon robot is equipped with a small piezo transducer and is programmed to continuously generate periodic chirps of predefined frequency. To generate a loud sound wave, that would result in a higher detection range of the 


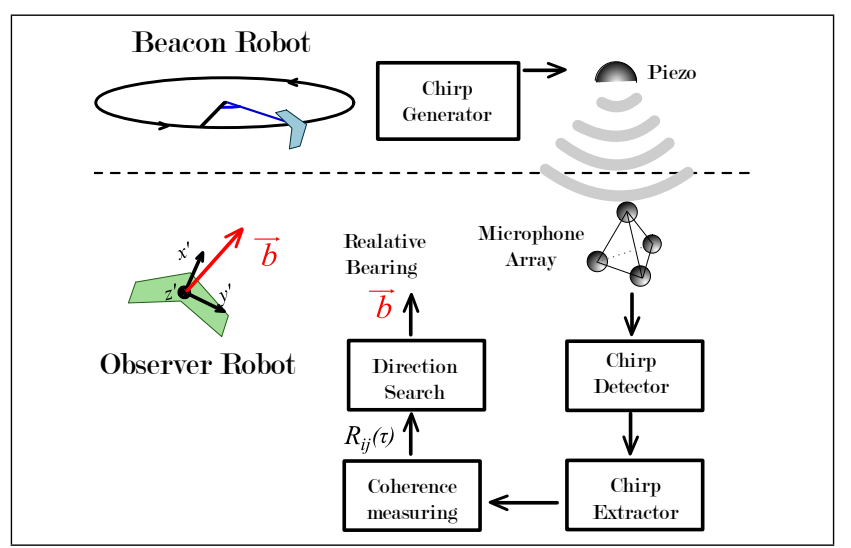

Fig. 2. Schematic diagram of the proposed Audio-based relative bearing measurement system illustrating the key parts of the system.

beacon MAV, a band-limited chirp with frequencies close to the resonance frequency of the piezo element is used. A chirp is used instead of a pure tone to avoid the problem of ambiguous measurements caused due to the repetitive nature of narrowband sounds. Figure 4(a) shows the frequency spectrogram of a single chirp, recorded in flight by an observer MAV, in presence of a chirping beacon MAV.

Observing MAVs are equipped with a microphone array, shown in figure 3 , to measure the sound waves at different points in space. An incoming chirp is picked up by the spatially separated microphones at different time instances. The existing delay between the microphone signals is used to measure the direction of arrival of the chirp. A minimum number of four microphones is needed to obtain the direction of arrival in 3D without ambiguity. Here, four microphones are used to minimize the hardware and computational loads. A tetrahedral microphone array geometry is used to obtain equivalent localization performance in all directions [20].

Microphone signals are continuously checked by the Chirp Detector for existence of the desired chirp in the sound mixture. The presence of a chirp is detected by template matching, where a template of the chirp, stored in the memory, is continuously cross-correlated with the signals. Upon detection of a chirp, the time window holding the entire chirp, for all microphones, is passed to the Chirp Extractor.

The Chirp Extractor filters the chirp from other sounds that might exist in the signal segment. For this, the time window containing the chirp is passed through a band pass filter to remove the unwanted low frequency wind noise and other high frequency noises (see Figure 4(b)). Furthermore,

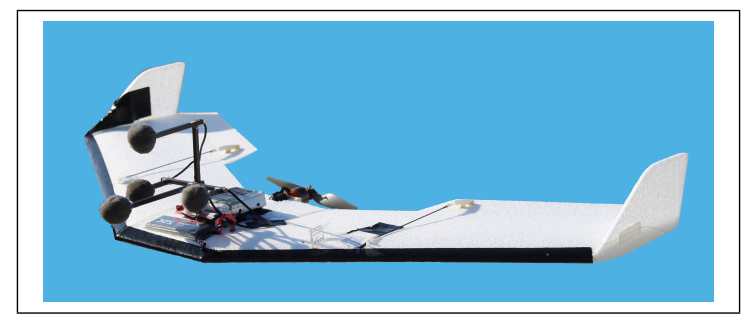

Fig. 3. Picture of the MAV platform [21] used in the experiments. A Microphone array of four microphones and a digital sound recorder is used for recording sounds during flight.

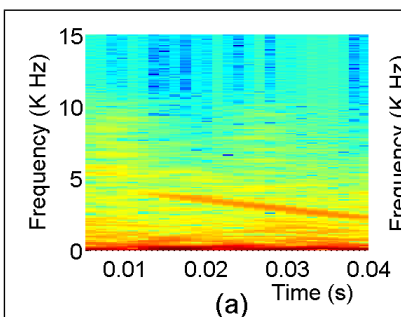

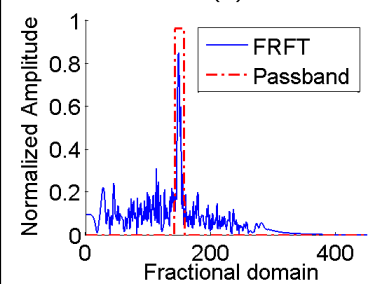

(c)

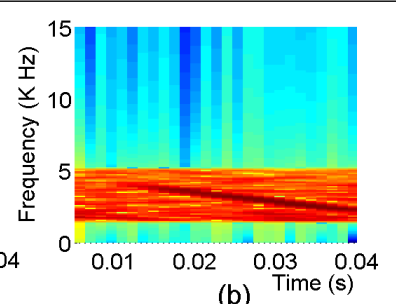

(b)

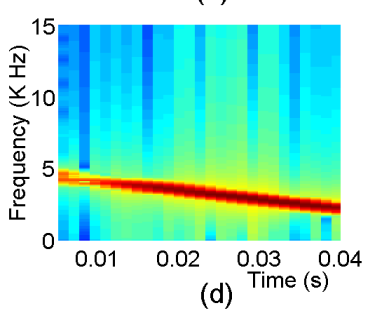

Fig. 4. In-flight sound of a chirping MAV recorded by an observing MAV. (a) Spectrogram of a detected chirp (b)Spectrogram of the signal after band-pass filtering (c) FRFT transform of the band-passed chirp and the corresponding passband region (d) Spectrogram of the final filtered chirp.

the Fractional Fourier transform (FRFT) [22] of the filtered signal is computed with a FRFT order of $\alpha$ obtained by:

$$
\alpha=\frac{2}{\pi} \tan ^{-1}\left(a \times f_{s}\right)
$$

where $f_{s}$ is the sampling frequency and $a$ is the chirp rate used by the beacon MAV. The computed FRFT contains a single impulse-shaped peak that corresponds to the chirp. The chirp is filtered out from other sounds that have made it through the band-pass filter, by only retaining the bin with the highest peak along with its few nearby bins and setting all other bins to zero (see Figure 4(c)). The ratio of the peak value to the mean value of all zeroed bins prior to zeroing provides a good measure for the quality of the perceived chirp. This measure is computed and used as a measure of reliability of the obtained bearing measurement and only measurements that satisfy a predefined reliability level are used in the update step of the EKF estimator. The filtered chirp in the FRFT domain is then transformed back to the time domain by computing the inverse FRFT.

After filtering, a measure of similarity between the chirps of every microphone pair is obtained by computing the inverse Fourier transform of the cross spectrum:

$$
R_{i j}(\tau)=\sum_{k=0}^{N-1} P_{i}[k] P_{j}^{*}[k] e^{i \frac{2 \pi k \tau}{N}}
$$

where $P_{i}(k)$ is the discrete Fourier transform of the signal of microphone $i, P_{j}^{*}$ denotes the complex conjugate of $P_{j}$ and $\tau$ is the correlation lag in samples in the range:

$$
-\frac{d_{m}}{c}<\tau<\frac{d_{m}}{c}
$$

where $d_{m}$ is the distance between the microphones and $c$ is the speed of sound.

A weighting function was introduced into equation (12) by [23] to whiten the cross-spectrum of the signals and allow equal contribution of all the frequencies in the cross correlation. Although this results in sharper cross correlation 


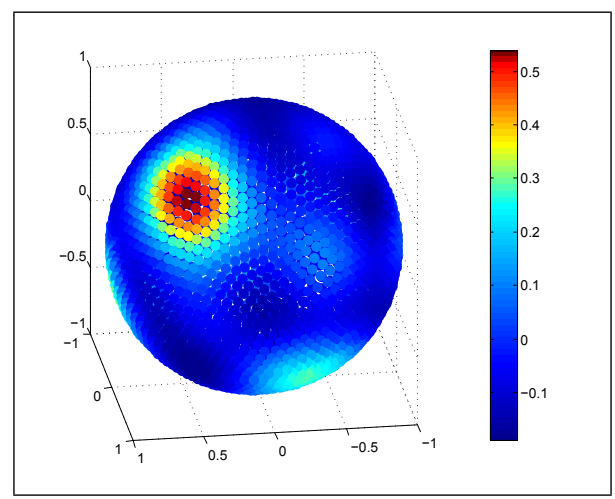

Fig. 5. Grid search of the in-flight chirp signal of Figure 4, showing the chirp's direction of arrival likelihood for all directions around the MAV. Each cell is on the surface of a unit sphere and represents the end of a unit vector starting from the origin that points towards a direction.

peaks for broadband sounds, it amplifies the background noise for narrowband sounds. A modified version of weighting function [23] is used here instead to only allow equal contribution of the desired frequencies:

$$
\begin{gathered}
R_{i j}(\tau)=\sum_{k=0}^{N-1} \chi\left[\frac{P_{i} P_{j}^{*}}{\left|P_{i}\right|\left|P_{j}\right|}\right] e^{i \frac{2 \pi k \tau}{N}} \\
\chi=\left\{\begin{array}{cc}
1 & f_{\min }<f<f_{\max } \\
0 & \text { otherwise }
\end{array}\right.
\end{gathered}
$$

where $f_{\min }$ and $f_{\max }$ are the minimum and maximum frequencies of the chirp.

Upon finding $R_{i j}$ from (13), for all microphone pairs $i j$, a search for the most likely source direction $\vec{b}_{m}$ is performed.

$$
\overrightarrow{b_{m}}=\underset{\vec{b}}{\arg \max } \sum_{i, j} R_{i j}\left(\tau_{\vec{b} i j}\right)
$$

where time delay $\tau_{\vec{b} i j}$ corresponds to direction $\vec{b}$ and is computed from the coordinates of microphones $i$ and $j$ in the body fixed coordinate system. In this work a full direction grid search, for a spherical geodesic grid of 2562 points, is performed. Other search methods for further reducing the cost of this search is available [24]. Figure 5 shows the result of a grid search for the perceived chirp described in Figure 4 illustrating the likelihood of all grid cells.

\section{EXPERIMENTS AND RESUlts}

To verify the bearing-only position estimator, described in section III-A, a group of MAVs were modelled using a computer simulation. Simulated MAVs were presented by a first order 3D flight model with three degrees of freedom for the airspeed, turn rate and the altitude, all controlled by PID controllers. The MAV's airspeed, turn rate and altitude dynamics have rate limitations and are influenced by a uniform noise. Furthermore, the sensors that provide the MAV's orientations, speed, altitude and the relative bearing to the beacon MAV were modelled to be affected by a zero mean uniform noise while the relative bearing sensor was limited in range. Model parameters were tuned to best represent a simple MAV platform that was

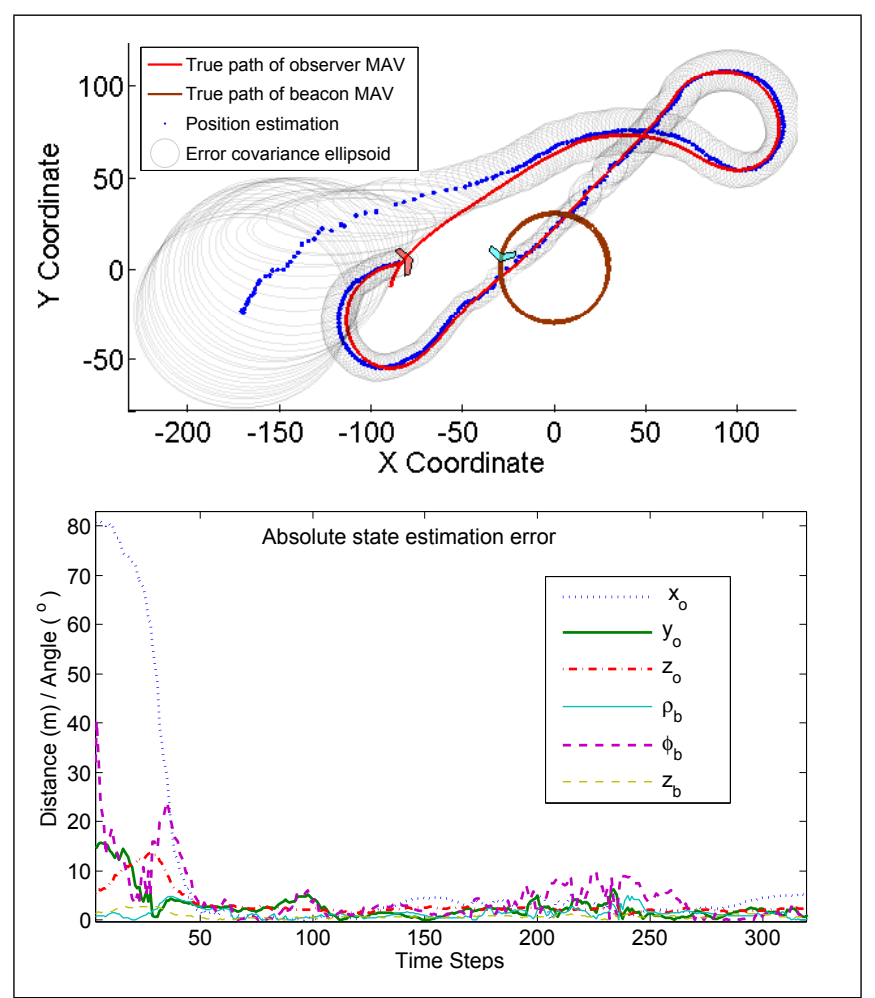

Fig. 6. Results of simulation with 1 observer and 1 beacon MAV. A uniform noise of $\pm 5^{\circ}$ for relative-bearing/attitude sensors and $\pm 1 \mathrm{~m}$ for altitude/speed sensors is used. The bearing sensor's detection range is $150 \mathrm{~m}$. top: Position estimates. bottom: Absolute estimation error of EKF states (1).

used throughout the real experiments [21]. A vector field controller is used to steer the motion of the MAVs onto a circular path around a point of interest. For a beacon robot this point is always static, while for an observer robot random points are generated sequentially to steer the robot between random points. Figure 6 shows the results of a simulation run involving a beacon MAV and an observer MAV. It shows the gradual convergence of the position estimations to the true position and the reduction in the error covariance.As expected, the convergence speed depends on the relative motion between the robots, where for the motions that result in a faster change of the relative bearing a faster localization is obtained. Upon localization, a good position tracking is achieved by the estimator.

Multiple real experiments were performed to verify the proposed audio-based relative bearing measurement system and the localization performance of the EKF estimator. A beacon MAV, equipped with an autopilot, was programmed to fly in circles around a GPS coordinate with constant velocity and constant altitude while emitting chirps using an on-board piezo. An observing MAV, shown in Figure 3, was then flown manually in proximity of the beacon MAV to record the sound waves using the on-board microphone array. The engine power of the observer MAV was occasionally reduced or turned off to increase the chirp to noise ratio and the detection range. The orientation, altitude, air-speed and global positioning information of both MAVs are measured using on-board sensors and are transmitted and stored on to 


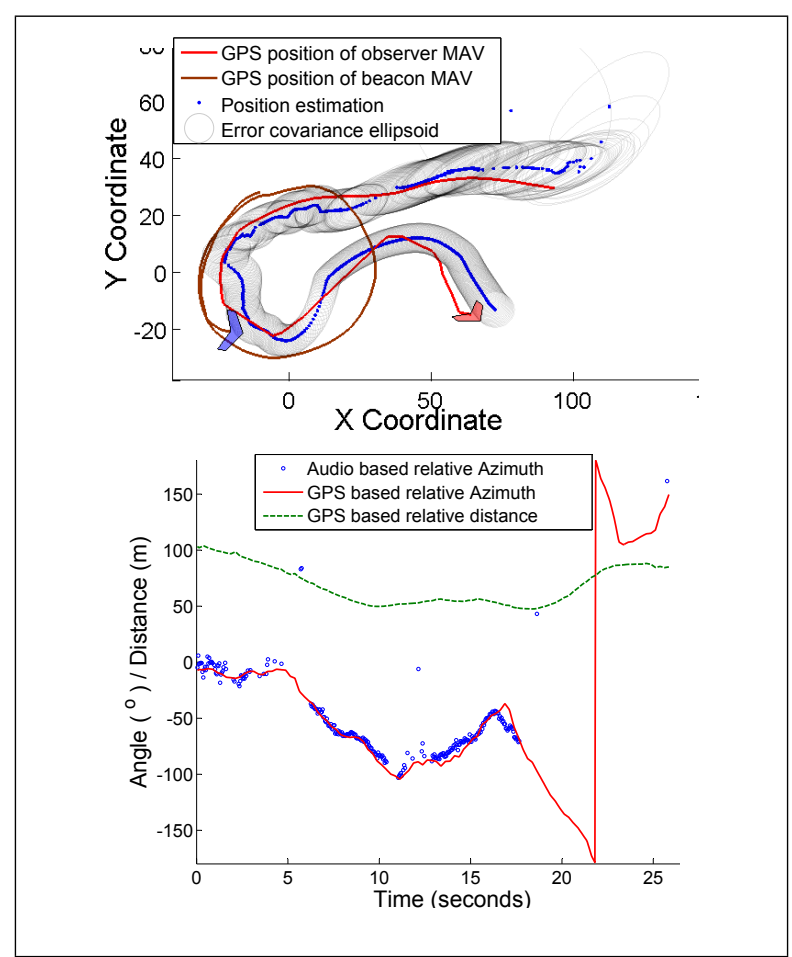

Fig. 7. Result of a real experiment with two MAVs. top: Audiobased position estimates and error covariance ellipsoid along with path of MAVs provided by GPS sensors. bottom: Audio-based relative bearing measurements along with the relative bearings and the relative distance between the robots obtained by the GPS sensors.

a ground station. Figure 7 shows the audio-based relative bearing measurements and the observer MAV's position estimates, along with a comparison against GPS based values, for an experiment. A good coherence between the GPS-based and audio-based estimates is observed. A root mean square error (rms) value of 6.9 degrees, as the error between the GPS-based and Audio-based bearing measurements, were obtained from 240 in-flight bearing measurements.

\section{CONClusion And Future WORK}

A solution to the problem of MAV swarm localization was presented. This solution consists of a single beacon MAV that circles around a reference point in space while emitting continuous linear chirps of predefined frequency spectrum to assist other MAVs in localizing themselves. MAVs are equipped with an on-board audio-based relative positioning system, to measure the bearing to the beacon MAV, and on-board sensors, to obtain information about their motions throughout time. The proposed EKF-based filter was shown to be well-suited for the sensor fusion and achieving a robust localization. No communication between the robots was required and only prior knowledge about robot's behaviour were used in the estimations. Investigating different types of MAV motions that could result in a faster localization, employing multiple beacon MAVs to improve localization performance, and study of switching protocols to switch MAVs between beacon and observer states, for exploration and reduction in the swarm's overall localization error, are some of the areas of work we are currently pursuing.

\section{REFERENCES}

[1] S. Hauert, S. Leven, J. Zufferey, and D. Floreano, "Communicationbased swarming for flying robots," in Proc. Intl. Conf. Robotics and Automation Workshop on Network Science and Systems, 2010.

[2] M. Basiri, F. Schill, P. Lima, and D. Floreano, "Robust acoustic source localization of emergency signals from micro air vehicles," in IEEE/RSJ International Conference on Intelligent Robots and Systems (IROS), oct. 2012, pp. $4737-4742$.

[3] D. T. Cole, A. H. Göktoan, and S. Sukkarieh, "The demonstration of a cooperative control architecture for uav teams," in Experimental Robotics. Springer, 2008, pp. 501-510.

[4] M. Basiri, A. Bishop, and P. Jensfelt, "Distributed control of triangular formations with angle-only constraints," Systems \& Control Letters, vol. 59, no. 2, pp. 147-154, 2010.

[5] N. Moshtagh, N. Michael, A. Jadbabaie, and K. Daniilidis, "Visionbased, distributed control laws for motion coordination of nonholonomic robots," IEEE Transactions on Robotics, vol. 25, no. 4, pp. 851 -860 , aug. 2009.

[6] R. Carnie, R. Walker, and P. Corke, "Image processing algorithms for uav "sense and avoid"," in IEEE International Conference on Robotics and Automation ICRA, may 2006, pp. 2848 -2853.

[7] A. Farnsworth, "Flight calls and their value for future ornithological studies and conservation research," The Auk, vol. 122, no. 3, 2005

[8] P. Muller and D. Robert, "A shot in the dark: the silent quest of a free-flying phonotactic fly," Journal of Experimental Biology, vol. 204, no. 6, pp. 1039-1052, 2001.

[9] M. Basiri, F. Schill, D. Floreano, and P. Lima, "Audio-based relative positioning system for multiple micro air vehicle systems," in Proceedings of Robotics: Science and Systems, Berlin, June 2013.

[10] G. M. Hoffmann, S. L. Waslander, and C. J. Tomlin, "Quadrotor helicopter trajectory tracking control," in AIAA Guidance, Navigation and Control Conference, Honolulu, Hawaii, 2008, pp. 1-14.

[11] L. Hu and D. Evans, "Localization for mobile sensor networks," in Proceedings of the 10th annual International Conference on Mobile computing and networking. ACM, 2004, pp. 45-57.

[12] R. Siegwart and I. Nourbakhsh, Introduction to autonomous mobile robots. MIT press, 2004.

[13] C. James et al., "Vulnerability assessment of the transportation infrastructure relying on the global positioning system," Volpe National Transportation Systems Center, US Department of Transportation, Tech. Rep, 2001.

[14] M. Blosch, S. Weiss, D. Scaramuzza, and R. Siegwart, "Vision based mav navigation in unknown and unstructured environments," in IEEE International Conference on Robotics and automation (ICRA), 2010.

[15] M. Bryson and S. Sukkarieh, "Building a robust implementation of bearing-only inertial slam for a uav," Journal of Field Robotics, vol. 24 no. 1-2, pp. 113-143, 2007.

[16] J. Artieda, J. M. Sebastian, P. Campoy, J. F. Correa, I. F. Mondragón, C. Martínez, and M. Olivares, "Visual 3-d slam from uavs," Journal of Intelligent and Robotic Systems, vol. 55, no. 4-5, pp. 299-321, 2009.

[17] E. W. Frew, D. A. Lawrence, C. Dixon, J. Elston, and W. J. Pisano, "Lyapunov guidance vector fields for unmanned aircraft applications," in American Control Conference. IEEE, 2007, pp. 371-376.

[18] D. R. Nelson, D. B. Barber, T. W. McLain, and R. W. Beard, "Vector field path following for miniature air vehicles," IEEE Transactions on Robotics, vol. 23, no. 3, pp. 519-529, 2007.

[19] B. Grocholsky, J. Keller, V. Kumar, and G. Pappas, "Cooperative air and ground surveillance," Robotics \& Automation Magazine, IEEE, vol. 13 , no. 3 , pp. $16-25,2006$.

[20] J.-S. Hu, C.-M. Tsai, C.-Y. Chan, and Y.-J. Chang, "Geometrical arrangement of microphone array for accuracy enhancement in sound source localization," in Control Conference (ASCC). IEEE, 2011.

[21] S. Leven, J. Zufferey, and D. Floreano, "A simple and robust fixedwing platform for outdoor flying robot experiments," in International symposium on flying insects and robots, 2007, pp. 69-70.

[22] V. Namias, "The fractional order fourier transform and its application to quantum mechanics," IMA Journal of Applied Mathematics, vol. 25, no. 3, pp. 241-265, 1980.

[23] C. Knapp and G. Carter, "The generalized correlation method for estimation of time delay," IEEE Transactions on Acoustics, Speech and Signal Processing, vol. 24, no. 4, pp. 320-327, 1976.

[24] J. Valin, F. Michaud, and J. Rouat, "Robust localization and tracking of simultaneous moving sound sources using beamforming and particle filtering," Robotics and Autonomous Systems, vol. 55, no. 3, pp. 216228, 2007. 